\section{Características, sintomas depressivos e fatores associados em mulheres encarceradas no Estado do Rio Grande do Sul, Brasil}

\author{
Characteristics, depressive symptoms, and \\ associated factors in incarcerated women \\ in the State of Rio Grande do Sul, Brazil
}

Daniela Canazaro 1

Irani Iracema de Lima Argimon 1

\footnotetext{
1 Pontifícia Universidade Católica do Rio Grande do Sul, Porto Alegre, Brasil.

Correspondência D. Canazaro Pontifícia Universidade Católica do Rio Grande do Sul. Rua Jari 619, apto. 1103, torre 3, Porto Alegre, RS 91530-001, Brasil.

danielacanazaro@hotmail.com
}

\begin{abstract}
The study's objective was to describe the socio-demographic and clinical characteristics of women inmates and verify the prevalence of depressive symptoms and hopelessness and drug and alcohol use, abuse, and addiction. The sample included 287 inmates at a Women's Penitentiary in the State of Rio Grande do Sul, Brasil. The study design was quantitative and cross-sectional. According to the findings, the typical female inmate was single, young, had at least two children, had worked in the informal market, and had low social and/or economic status and incomplete primary schooling. There was a high prevalence of depressive symptoms and drug use, abuse, and addiction, but a low prevalence of symptoms of hopelessness. Various factors, especially linked to the participants' life history, were significantly associated with depressive symptoms.
\end{abstract}

Prisoners; Women; Depression; Street Drugs

\section{Introdução}

Os altos índices de violência e criminalidade em nossa sociedade refletem no aumento de prisioneiros. A literatura aponta diversos modelos para descrever as causas desses fenômenos, nos quais, cada teoria, centra-se em alguns fatores em particular, focando desde as patologias individuais até as sociais. As primeiras, individuais, atualmente estão sendo relacionadas às características biopsicológicas, juntamente com o histórico de vida pessoal e com as relações sociais, englobando assim outros fatores associados 1. Dessa forma, os estudiosos apontam diversos e diferentes fatores relacionados à criminalidade, descrevendo-a como um fenômeno complexo que abrange fatores biológicos, genéticos, psicológicos, econômicos, culturais etc. Pesquisas apontam uma alta prevalência de transtornos mentais e comorbidades da população carcerária 2 , o que pode ser entendido como um problema de saúde pública mundial. As doenças mentais estão relacionadas com diversos fatores, tais como baixo nível socioeconômico, desemprego, história de abuso sexual ou físico, uso de substâncias psicoativas 3,4, bem como história familiar de problemas mentais e de dependência química, dentre outros 5 .

O Brasil está em quarto lugar no ranking dos países com maior população carcerária (422.590, sendo 403.556 homens e 19.034 mulheres). Os primeiros países são: Estados Unidos (2.299.116 presos), China (1.565.771) e Rússia (894.855) 
(International Center for Prison Studies. Prison brief: highest to lowest rates. http://www.kcl. ac.uk/depsta/law/research/icps/worldbrief/ wpb_stats.php?area=all\&category=wb_poptotal, acessado em 20/Set/2008).

No Brasil, nos últimos quatro anos, houve um aumento da população carcerária feminina de $37,47 \%$, representando um crescimento anual de $11,99 \%$. Em verdade, o aumento da população feminina tem sido maior se comparado com o masculino. Estima-se que no ano de 2012 a população feminina representará $7,65 \%$, enquanto que atualmente representa apenas $6,12 \%$ no país. Outro dado relevante, que merece destaque, é o fato de a população carcerária no Brasil, assim considerados tanto os homens como as mulheres, em sete anos duplicou, enquanto que no Estado do Rio Grande do Sul a população carcerária feminina praticamente triplicou, passando de 377 para 1.060 reclusas (Departamento de Segurança e Execução Penal, Superintendência dos Serviços Penitenciários. Rio Grande do Sul: mapa carcerário; 2007. http://www.susepe.rs.gov.br, acessado em 16/Out/2007).

Estudos consideram que os prisioneiros possuem taxas mais elevadas de problemas mentais, quando comparados com a comunidade em geral 6,7. Dados estatísticos apontam que a prevalência de doença mental na comunidade está no porcentual de $15 \%$, enquanto que entre a população carcerária o percentual é de $42 \% 8$. Evidencia-se, assim, uma alta prevalência de transtornos mentais nos prisioneiros, variando de $37 \%$ a $89 \% 9$.

As pesquisas descrevem que as presas têm um elevado grau de comorbidade psicopatológica, dependência de substância, transtorno de estresse pós-traumático e depressão maior. As encarceradas são também mais propensas a apresentar doenças mentais, quando comparadas com a população carcerária masculina, exceto quando se trata de transtorno de personalidade anti-social ${ }^{10}$. Observa-se que de um a dois terços de todas essas mulheres necessitam de tratamento mental e, aproximadamente, um quinto tem uma história de uso de medicação psicotrópica 11. Além disso, apresentam maiores experiências traumáticas, incluindo abuso físico e sexual precoce. Nesse sentido, o estudo de Johnson 4 apontou altas taxas de abuso entre as mulheres, totalizando $87 \%$ da amostra. A prevalência de abuso e dependência de substância variou de $10 \%$ a $48 \%$ nos presos homens e de $30 \%$ a $60 \%$ nas mulheres presas 12 ; a prevalência de depressão maior foi verificada em $10 \%$ dos homens e $12 \%$ nas mulheres encarceradas 13 .

As mulheres prisioneiras são diferentes dos homens prisioneiros por diversos motivos, den- tre os quais se podem destacar: (a) o padrão dos crimes das mulheres impõe um menor nível de risco à comunidade; (b) é provável que as mulheres sejam mais responsáveis pelo cuidado dos filhos e pela manutenção da casa do que os homens, de modo que o impacto da prisão é desproporcionalmente mais grave para as prisioneiras, freqüentemente resultando na perda do lar e em dano grave na vida de seus filhos; (c) a mulher sofre uma cadeia mais grave de exclusão social do que o homem, apresentando altos níveis de abuso e violência doméstica e problema de saúde mental. A prisão é conhecida por ter implicações psicológicas graves para mulheres, sendo comuns comportamentos autodestrutivos em todas as prisões femininas 14 . Covington 15, também ressaltando a diferença, aponta que a mulher tem maior risco de ingressar na prisão com doenças sexualmente transmissíveis, como HIV/AIDS, por causa de seu maior envolvimento com a prostituição e história de abuso sexual.

A conduta criminosa pode estar associada a diversos fatores, como já ressaltado anteriormente, sejam eles, biológicos, genéticos, econômicos, culturais e, psicológicos, dentre outros. Neste estudo, serão abordados os aspectos psicológicos, enfocando a identificação das características sociodemográficas e clínicas; a prevalência de sintomas depressivos e de desesperança; o uso, abuso e/ou dependência de drogas em mulheres encarceradas.

Com base nos resultados, busca-se descrever as mulheres que compõem a população carcerária, além de estabelecer um início de reflexão, fornecendo elementos para futuras intervenções no sistema prisional e elaboração de políticas públicas de saúde.

\section{Métodos}

Trata-se de um estudo com delineamento transversal, descritivo e quantitativo. A amostra foi constituída por 287 mulheres, todas recolhidas na Penitenciária Feminina Madre Pelletier, na cidade de Porto Alegre, Rio Grande do Sul. Essa é a única penitenciária de regime fechado exclusiva para mulheres no estado. No mês de dezembro de 2007, a instituição estava constituída por 368 mulheres, as quais representavam aproximadamente $35 \%$ da população feminina de prisioneiras totais do estado.

A equipe, previamente treinada, constituiuse por três auxiliares de pesquisa (estudantes de psicologia) e duas psicólogas que trabalham na Casa Prisional, sendo uma delas a responsável pela pesquisa, a qual capacitou os entrevista- 
dores. Os cinco integrantes aplicaram todos os questionários.

As entrevistas foram individuais e tiveram, em média, uma hora de duração. Como instrumentos foram utilizados, na seguinte ordem: (a) ficha de dados sociodemográficos e clínicos (nível de escolaridade, estado civil, tempo de reclusão, situação jurídica, história de tratamento psiquiátrico antes e durante o aprisionamento, uso de drogas e história penal pessoal e familiar); (b) questionário CAGE; (c) Entrevista Clínica Estruturada para o DSM-IV - Versão Clínica (SCID-CV) - módulo de transtorno do uso de substância não-alcoólica; (d) Inventário de Depressão de Beck (BDI II); (e) Escala de Desesperança de Beck (BHS).

A coleta dos dados ocorreu nos meses de dezembro de 2007 e janeiro de 2008. Todas as mulheres recolhidas na penitenciária referida foram chamadas individualmente nas salas de atendimento psicológico, com o intuito de esclarecer o objetivo e a metodologia da pesquisa a cada participante. No início da coleta, a população prisional era constituída por 368 mulheres. Durante os dois meses de coleta, 57 encarceradas saíram dessa instituição prisional, seja porque receberam liberdade provisória, habeas corpus, revogação da prisão preventiva, absolvição ou transferência para outra instituição de regime fechado ou semi-aberto. Somente 23 presas não aceitaram participar da pesquisa - algumas delas nem chegaram a se deslocar para a entrevista, recusando-se a sair da cela. Havia, ainda, uma de castigo. Ao final, 287 mulheres aceitaram participar, representando $80 \%$ do universo de encarceradas nessa penitenciária.

A amostra foi descrita estatisticamente em suas características sociodemográficas e clínicas, mediante análise das distribuições de freqüência das variáveis dependentes e independentes. Realizou-se uma análise bivariada dos resultados obtidos por meio dos testes qui-quadrado, $t$ de Student, regressão logística bivariada e multivariada. Foram consideradas significativas as associações com valores de $\mathrm{p}<0,05$. Calcularam-se as estatísticas usando-se o SPSS versão 11.0 para Windows (SPSS Inc., Chicago, Estados Unidos).

Esta pesquisa foi autorizada pela direção da penitenciária e pelo órgão ao qual está subordinada, a Superintendência dos Serviços Penitenciários (SUSEPE). Após, o projeto foi aprovado pela Comissão Científica da Faculdade de Psicologia da Pontifícia Universidade Católica Rio Grande do Sul, onde este estudo foi desenvolvido. Conforme esse parecer, o estudo foi autorizado sem a necessidade de passar pelo Comitê de Ética, devido características específicas desta pesquisa. Este estudo faz parte de outro projeto de pesquisa maior, associado com a validação de uma escala (BDI II), e este projeto já havia sido aprovado pelo Comitê de Ética.

A entrevista constituiu-se em uma sessão com cada participante, na qual, após informar o objetivo do estudo, foi assinado o Termo de Consentimento Livre e Esclarecido. Todas as entrevistadas aceitaram voluntariamente participar desta atividade, sendo a elas esclarecido que a decisão em não participar não implicaria nenhum prejuízo, sobretudo no que se referia à situação jurídica.

\section{Resultados}

\section{Dados sociodemográficos}

Quanto à idade, as prisioneiras apresentaram uma média de 33,39 anos (desvio-padrão - DP = 9,84), sendo 18 anos a idade mínima e 61 a máxima. No quesito estado civil, 46,7\% são solteiras; se somadas as viúvas, as separadas e as divorciadas, resulta no porcentual de $67,6 \%$ que não têm companheiros. Do total, 86,4\% têm filhos, com uma média de 2,75 filhos. Quanto à escolaridade, a maioria tem no máximo o Ensino Fundamental incompleto, representado por $63,1 \%$.

Quanto a atividades laborativas, 75,2\% das mulheres estavam trabalhando antes do encarceramento, porém apenas $15,9 \%$ tinham um emprego formal. Uma grande parcela tem uma profissão de baixo status social e/ou econômico, como empregada doméstica/faxineira $(27,9 \%)$, promotora de vendas/vendedora $(15,7 \%)$, donade-casa $(13,6 \%)$, profissional do sexo $(6,3 \%)$, sem profissão $(5,9 \%)$, auxiliar administrativo/produção $(5,6 \%)$ e outras.

\section{Dados criminais e prisionais}

A amostra é constituída por $51,2 \%$ de presas provisórias - as que aguardam decisão da Justiça a respeito do processo criminal pelo qual estão respondendo - e $48,8 \%$ de presas condenadas. Um porcentual de $34,8 \%$ das mulheres já havia sido recolhido à prisão. Mais da metade da amostra (51,6\%) está reclusa no máximo até seis meses, o que indica que, provavelmente, muitas devem ser provisórias. Com relação ao delito praticado, a maioria responde por tráfico de drogas $(62,4 \%)$, seguido de roubo $(12,5 \%)$, homicídio $(11,1 \%)$ e furto $(8,7 \%)$.

Com relação ao aprisionamento, $51,2 \%$ estavam trabalhando e $15,3 \%$ estudavam no momento da coleta de dados. Grande parte recebe visita de familiares e/ou amigos, representada por $77,4 \%$. Porém, apenas uma minoria recebe 
visita íntima (espaço privado para relações sexuais), totalizando $8,5 \%$ da amostra.

\section{Prevalência de problemas de saúde mental}

A Tabela 1 mostra fatores de prevalência relacionados aos problemas de saúde mental. A prevalência de uso de drogas e álcool ao longo da vida é elevada (54,4\% e 53,7\%), representada pela metade da amostra de mulheres encarceradas. Das participantes, $15,7 \%$ desenvolveram dependência de álcool e 38,3\% dependem de outras drogas. As mais utilizadas são: maconha (47\%), cocaína $(38,3 \%)$ e $\operatorname{crack}(27,2)$.

A Tabela 2 mostra alguns fatores que podem estar associados com sintomas depressivos (como fuga de casa, ocorrência de abuso não-sexual ou sexual, familiares com problemas psiquiátricos ou envolvidos com o uso de drogas e/ou álcool), que provavelmente foram desencadeados quando as encarceradas se encontravam em liberdade. Evidencia-se uma alta prevalência de mulheres que relatam problemas de saúde física e psicológica, e história de ideação suicida ao longo da vida, bem como relato de tratamento psicológico quando em liberdade.

Também se evidencia alta prevalência de mulheres que possuem familiares com história de uso de drogas e/ou álcool e com problemas psiquiátricos, o que pode estar relacionado à história de violência e ocorrência de fuga de casa. Provavelmente, essas mulheres precocemente passaram por situações de risco e tiveram contato com drogas dentro do ambiente familiar ou em situação de rua.

A respeito da história de internação, do tratamento psiquiátrico e da tentativa de suicídio,

Tabela 1

Percentil de problemas de saúde mental das mulheres presas. Porto Alegre, Rio Grande do Sul, Brasil, 2008.

\begin{tabular}{lcc}
\hline Variáveis & $\mathbf{n}$ & $\%$ \\
\hline Uso de drogas (ao longo da vida) & 156 & 54,4 \\
Uso de álcool & 154 & 53,7 \\
Sintomas de depressão & 140 & 48,7 \\
Abuso de drogas & 113 & 39,4 \\
Dependência de drogas & 110 & 38,3 \\
Uso atual de drogas (até um ano) & 78 & 27,2 \\
Uso passado de drogas (mais de um ano) & 52 & 18,1 \\
Dependência de álcool & 45 & 15,7 \\
Sintomas de desesperança & 17 & 5,9 \\
Total & 287 & 100,0 \\
\hline
\end{tabular}

evidencia-se que a maior ocorrência foi antes do aprisionamento. Em liberdade, 17,1\% das prisioneiras já tinham realizado tratamento psiquiátrico, e 9,1\% fizeram tratamento na prisão. A internação psiquiátrica de 9,4\% ocorreu ainda em liberdade e $1 \%$ quando as encarceradas já estavam presas. Outro dado importante é que 13,6\% das participantes já tinham história pregressa de tentativa de suicídio e $4,2 \%$ apresentaram a tentativa durante o aprisionamento.

Tempo de reclusão e situação jurídica relacionados com sintomas depressivos

Foi realizada uma análise de associação entre a presença de sintomas de depressão e o tempo de reclusão. Os resultados mostram, conforme a Tabela 3, que não existe uma associação significativa entre o tempo de reclusão e a intensidade dos sintomas depressivos. Os sintomas depressivos não aumentaram com o tempo de reclusão, antes pelo contrário, eles são menos freqüentes após 26 meses de prisão. Esse achado sugere que o tempo em que a mulher permanece presa não interfere na intensidade dos sintomas depressivos. Com relação à situação jurídica, a presença dos sintomas depressivos também não se mostrou diferente entre as provisórias e as já condenadas.

\section{Associação entre variáveis e sintomas depressivos}

Baseando-se nas características apresentadas pelas participantes foi possível realizar algumas associações com a presença de sintomatologia depressiva. A Tabela 4 mostra relações entre as variáveis e a média total do BDI II por meio do teste t, o qual possibilita realizar associações entre as variáveis e a média dos escores brutos.

Pode-se observar que a média significativamente mais elevada de sintomatologia depressiva está entre as mulheres que apresentam os seguintes históricos: ideação e/ou tentativa de suicídio; tratamento e/ou internação psiquiátrica; uso de medicação psiquiátrica sem prescrição durante o aprisionamento; percepção de que têm algum problema psicológico; grupo familiar com problemas psiquiátricos; sofreram violência; uso de drogas e/ou álcool. O percentual dentre essas mulheres é maior quando comparadas com outras que não passaram por tais situações.

Um dado importante é que as participantes que trabalham na prisão apresentam menores sintomas depressivos, sendo que os dados são significativos. Das encarceradas que trabalham, 91,9\% afirmaram sentirem-se satisfeitas com sua atividade laborativa, parecendo ser um fator de 
Características clínicas das mulheres presas. Porto Alegre, Rio Grande do Sul, Brasil, 2008.

\begin{tabular}{llc}
\hline Variáveis & $\mathbf{n}$ & $\%$ \\
\hline Familiar usa/usou drogas e/ou álcool & 209 & 72,8 \\
Percepção de que tem algum problema de saúde física & 148 & 48,1 \\
Ocorrência ou tentativa de fuga de casa & 118 & 41,1 \\
Ideação suicida (ao longo da vida) & 114 & 39,7 \\
Familiar com problemas psiquiátricos & 93 & 32,4 \\
Ocorrência de violência não-sexual & 90 & 31,4 \\
Uso de medicação psiquiátrica na prisão atual & 87 & 30,3 \\
Percepção de que tem algum problema psicológico & 67 & 23,3 \\
Tratamento psicológico em liberdade & 58 & 20,2 \\
Uso de medicação psiquiátrica na prisão sem prescrição atual & 49 & 17,1 \\
Ocorrência de violência sexual & 49 & 17,1 \\
Total & 287 & 100,0 \\
\hline
\end{tabular}

Tabela 3

Associação entre sintomas depressivos com tempo de reclusão e situação jurídica. Porto Alegre, Rio Grande do Sul, Brasil, 2008

\begin{tabular}{|c|c|c|c|c|c|c|c|}
\hline \multirow[t]{2}{*}{ Variáveis } & \multicolumn{2}{|c|}{$\begin{array}{c}\text { Sem sintomas } \\
(n=144)\end{array}$} & \multicolumn{2}{|c|}{$\begin{array}{l}\text { Com sintomas } \\
\qquad(n=140)\end{array}$} & \multicolumn{2}{|c|}{ Total } & \multirow[t]{2}{*}{ Valor de $p$} \\
\hline & $\mathrm{n}$ & $\%$ & $n$ & $\%$ & $\mathrm{n}$ & $\%$ & \\
\hline \multicolumn{8}{|c|}{ Tempo de reclusão (meses) } \\
\hline Até 6 & 76 & 52,1 & 70 & 47,9 & 146 & 100,0 & 0,471 \\
\hline $7-12$ & 33 & 45,2 & 40 & 54,8 & 73 & 100,0 & \\
\hline $12-14$ & 21 & 48,8 & 22 & 51,2 & 43 & 100,0 & \\
\hline Acima de 26 & 14 & 63,6 & 8 & 36,4 & 22 & 100,0 & \\
\hline \multicolumn{8}{|l|}{ Situação jurídica } \\
\hline Provisória & 75 & 52,4 & 71 & 48,6 & 145 & 100,0 & 0,911 \\
\hline Condenada & 69 & 50,0 & 69 & 50,0 & 138 & 100,0 & \\
\hline
\end{tabular}

proteção para a minimização de sintomas depressivos durante a execução da pena. Porém, quem estuda na prisão não teve diferença significativa nas médias.

Observa-se que algumas características não irão influenciar de modo significativo nas médias apontadas, dentre elas, cumpre destacar: se a presa recebe visita; se há pena condenatória; se já visitou alguém na prisão; se possui familiar com problemas de drogas e/ou álcool; se tem familiar que já cumpriu pena. Já as participantes que são mães apresentam uma tendência de ter uma média maior de sintomas depressivos do que as que não têm filhos.
Foi realizada uma regressão logística com relação à sintomatologia depressiva, verificando-se a influência de variáveis independentes, ou seja, a probabilidade de ocorrência de certos eventos.

Observou-se que algumas variáveis independentes podem ser consideradas fatores de risco por aumentarem a chance de desenvolver a sintomatologia depressiva grave. Os fatores que constam na Tabela 5 são: ideação suicida, percepção de possuir um problema psicológico, história de tratamento psiquiátrico, tentativa de suicídio, familiares com problemas psiquiátricos e história de violência não-sexual aumentam significativamente a probabilidade da ocorrência 
Associação entre variáveis e a média total dos sintomas depressivos. Porto Alegre, Rio Grande do Sul, Brasil, 2008.

\begin{tabular}{|c|c|c|c|c|c|}
\hline \multirow[t]{2}{*}{ Variáveis } & \multicolumn{2}{|c|}{ Não } & \multicolumn{2}{|c|}{ Sim } & \multirow[t]{2}{*}{ Valor de $p$} \\
\hline & Média & DP & Média & DP & \\
\hline Ideação suicida & 17,8 & 9,28 & 27,9 & 12,36 & $<0,001$ \\
\hline Tentativa de suicídio & 19,6 & 10,14 & 30,0 & 13,56 & $<0,001$ \\
\hline Tratamento psiquiátrico & 19,3 & 10,42 & 27,4 & 12,48 & $<0,001$ \\
\hline Percepção de problema psicológico & 19,5 & 10,42 & 29,3 & 12,60 & $<0,001$ \\
\hline Medicação sem prescrição & 20,0 & 10,59 & 30,8 & 13,00 & $<0,001$ \\
\hline Uso de drogas (ao longo da vida) & 18,9 & 10,62 & 24,2 & 12,05 & $<0,001$ \\
\hline Familiar com problemas psiquiátricos & 20,1 & 11,20 & 25,2 & 12,05 & 0,001 \\
\hline Internação psiquiátrica & 20,7 & 10,87 & 30,1 & 14,44 & 0,001 \\
\hline Violência não-sexual & 20,4 & 11,24 & 24,3 & 11,96 & 0,008 \\
\hline Está trabalhando & 23,4 & 12,77 & 20,2 & 10,36 & 0,021 \\
\hline Uso de álcool & 19,6 & 11,09 & 23,7 & 11,92 & 0,003 \\
\hline Violência sexual & 21,0 & 11,55 & 24,7 & 11,83 & 0,048 \\
\hline Filhos & 18,4 & 11,60 & 22,3 & 11,65 & 0,052 \\
\hline Recebe visita & 24,0 & 12,52 & 21,1 & 11,39 & 0,081 \\
\hline Familiar com história drogas/álcool & 20,1 & 10,99 & 22,4 & 11,92 & 0,138 \\
\hline Está estudando & 21,4 & 11,64 & 23,9 & 12,33 & 0,191 \\
\hline Já visitou alguém na prisão & 20,9 & 11,52 & 22,4 & 11,84 & 0,287 \\
\hline Situação jurídica (condenada) & 22,3 & 11,60 & 21,3 & 11,82 & 0,480 \\
\hline Familiar já cumpriu pena & 21,2 & 11,54 & 22,1 & 11,79 & 0,522 \\
\hline
\end{tabular}

Tabela 5

Análise de regressão logística bivariada entre as variáveis e a sintomatologia depressiva: variável resposta. Porto Alegre, Rio Grande do Sul, Brasil, 2008.

\begin{tabular}{llc}
\hline Variáveis independentes & OR (IC95\%) & Valor de p \\
\hline Ideação suicida & $3,88(2,34-6,44)$ & $<0,001$ \\
Percepção de problema psicológico & $3,65(1,99-6,69)$ & $<0,001$ \\
Tratamento psiquiátrico & $3,11(1,83-5,29)$ & $<0,001$ \\
Tentativa de suicídio & $2,90(1,57-5,35)$ & 0,001 \\
Familiar com problema psiquiátrico & $2,02(1,22-3,36)$ & 0,006 \\
Violência não-sexual & $1,88(1,13-3,13)$ & 0,020 \\
Violência sexual & $1,73(0,93-3,30)$ & 0,080 \\
Está trabalhando & $0,76(0,47-1,20)$ & 0,237 \\
Familiar já cumpriu/cumpre pena & $1,28(0,78-2,10)$ & 0,331 \\
Já visitou alguém na prisão & $1,20(0,74-1,93)$ & 0,464 \\
\hline
\end{tabular}

de sintomatologia grave. Assim, quem sofreu violência não-sexual tem 0,88 mais chance de apresentar sintomatologia depressiva quando comparado com quem não sofreu. As participantes cujos familiares apresentam problemas psiquiátricos têm uma vez mais chance de desenvolver a sintomatologia depressiva do que as que não possuem familiares com tal característica. Quem apresenta história de ideação e tentativa de suicídio tem, respectivamente, 2,88 e 1,90 mais chances de apresentar a sintomatologia depressiva. As presas que percebem ter um problema psicológi- 
co têm 2,56 mais chances de apresentar sintomatologia depressiva. As com história de tratamento psiquiátrico têm 2,11 mais chances de desenvolver sintomatologia depressiva.

Já o contato anterior com o ambiente prisional - por meio de familiares que cumprem ou cumpriram pena, ou das encarceradas que já visitaram alguém na prisão - não está relacionado ao desencadeamento de tais sintomas (Tabela 5).

$\mathrm{Na}$ análise multivariada foram incluídas todas as variáveis significativas na análise bivariada com $\mathrm{p}<0,15$, sendo que o modelo final foi relacionado à variável resposta depressão, à ideação suicida, ao tratamento psiquiátrico e ao tempo de reclusão. Constatou-se que as detentas que apresentam ideação suicida têm 2,80 mais chances de apresentar sintomatologia depressiva grave do que as que não têm tal ideação. Quem tem história de tratamento psiquiátrico possui aproximadamente uma chance a mais de ter sintomatologia depressiva grave, em comparação com as mulheres que não fizeram tratamento psiquiátrico. Ainda, quem está encarcerada há mais de 25 meses apresenta 6,88 menos chances de desenvolver a sintomatologia depressiva do que quem está até seis meses presa. O maior tempo de reclusão torna-se um fator de proteção (Tabela 6).

Tabela 6

Análise de regressão logística multivariada: ideação suicida, tratamento psiquiátrico e tempo de reclusão. Porto Alegre, Rio Grande do Sul, Brasil, 2008.

\begin{tabular}{lrc}
\hline Variáveis independentes & OR (IC95\%) & $\begin{array}{r}\text { Valor } \\
\text { de p }\end{array}$ \\
\hline Ideação suicida & $3,80(2,13-6,76)$ & $<0,001$ \\
Tratamento psiquiátrico & $1,98(1,10-3,58)$ & 0,023 \\
Tempo até 6 meses & 1,00 & 0,052 \\
Tempo de 7-12 meses & $1,52(0,89-2,80)$ & 0,182 \\
Tempo de 12-24 meses & $1,10(0,53-2,32)$ & 0,794 \\
Tempo acima de 25 meses & $0,32(0,12-0,90)$ & 0,030 \\
\hline
\end{tabular}

\section{Discussão}

Diante do aumento de mulheres envolvidas com a criminalidade e a conseqüente elevação do número da população carcerária feminina, é prudente descrever as características e os fatores da trajetória de vida das presas.
Por meio de algumas peculiaridades da mulher presa, descritas neste estudo, pode-se supor que ela está em uma posição social e econômica desfavorável. Embora uma grande parte das mulheres estivesse trabalhando antes da detenção, a maioria possui o Ensino Fundamental incompleto, baixa qualificação profissional, atividade laborativa informal e trabalhos de baixa rentabilidade. Além dessas características, muitas possuem um elevado número de filhos e são solteiras, provavelmente desempenhando o papel de provedoras e de cuidadoras da família. Estudos específicos com a população carcerária confirmam esses achados, apontando que grande parte da amostra de mulheres encarceradas vem das camadas mais baixas da sociedade, evidenciada pela baixa escolaridade e atividades laborais de pouca qualificação e salários proporcionais, e por histórico de vida marcado por insuficiência material, educacional e afetiva 16 .

A alta prevalência de sintomatologia grave de depressão, entre as encarceradas, objeto do presente estudo, representa quase a metade da amostra $(48,7 \%)$. Esse mesmo alto percentual foi encontrado em outro estudo 17, o qual denota que $61,7 \%$ das mulheres reclusas apresentaram depressão ao longo da vida. Já outra pesquisa, em que foi aplicado o BDI II, apontou $18 \%$ de prevalência de sintomas depressivos clinicamente significativos e $68,6 \%$ apresentando disforia 18.

Os resultados do presente estudo mostram que mais da metade da amostra tem história de uso de álcool e 15,7\% apresentam dependência. Estudos apontam uma elevada porcentagem nos prisioneiros, sendo encontrada uma diferença significativa na prevalência de transtorno relacionado ao uso de álcool maior entre os homens $7,19,20$. Os percentuais apurados foram $46,7 \%$ para os homens e $26,5 \%$ para as mulheres 21 .

Constatam-se também altas taxas no uso de outras drogas. Foi apurado que mais da metade da amostra pesquisada tinha história de uso de drogas, sendo que grande parte fechou os critérios para dependência, representada por 38,3\% das encarceradas. Essas informações são corroboradas pelos estudos de Belenko \& Peught 22, o qual aponta que $70 \%$ das mulheres presas fazem uso regular de drogas.

Não obstante as mulheres presas apresentem uma intensidade alta de sintomas depressivos no momento atual, o presente estudo mostra que a sintomatologia de desesperança, ou seja, a prevalência da extensão das expectativas negativas a respeito do futuro imediato e remoto, é extremamente menor. Apenas 5,9\% apresentam uma visão negativa para o futuro. A ausência de sintomas de desesperança, por meio de perspectiva 
positiva frente ao futuro, pode ser vista como um fator de esperança quanto ao futuro e proteção para permanecer certo tempo na prisão. Observase, ainda, que as encarceradas demonstram uma percepção positiva frente ao futuro, o que indica menor risco de suicídio, mesmo que a ideação possa estar mais representada pelo grau da gravidade dos sintomas depressivos. Esse resultado é corroborado por um estudo 23 que menciona alta prevalência de comportamento relacionado com o suicídio $(40,8 \%)$ e tendo uma diferença significativa maior entre as mulheres do que entre os homens reclusos. Porém, o ato suicida, caracterizado pela letalidade de comportamento relativo ao suicídio (risco de suicídio), foi significativamente menor para as mulheres do que para os homens.

Em princípio, a elevada sintomatologia depressiva manifestada pelas mulheres poderia estar ligada ao aprisionamento, que causa impacto com a privação de liberdade e ruptura dos laços afetivos. Todavia, não houve diferença significativa na presença de sintomas, se comparadas as mulheres encarceradas provisórias com aquelas já condenadas. Também não houve diferença na sintomatologia depressiva, se considerado o fator tempo de reclusão. Com base nessas informações, pode-se entender que os fatores pessoais contribuem para o desencadeamento de tal sintomatologia.

Foi apurado que uma minoria recebe visita íntima, mas que a maioria recebe visita de familiares e/ou amigos. Supõe-se, dessa forma, que o suporte externo representa um fator importante para evitar a intensificação dos sintomas depressivos ao longo do tempo de reclusão. Mesmo não sendo significativa a diferença, as mulheres que recebem visita têm uma média menor da sintomatologia depressiva. Além disso, quem trabalha na prisão apresenta uma média significativamente menor de sintomatologia depressiva, comparando-se com quem não trabalha. Ao contrário, as mulheres que estudam, além de não apresentarem diferenças significativas, demonstram uma média maior dos sintomas depressivos.

A análise multivariada constatou que a presença de ideação suicida e tratamento psiquiátrico e o tempo de aprisionamento aumentaram a probabilidade de desencadear a sintomatologia depressiva. Em contraste, as confinadas que estão há mais de 25 meses apresentam uma menor probabilidade de desenvolver tal sintomatologia, tornando-se então um fator de proteção. Esses dados reforçam que há uma diversidade de fatores que podem estar associados aos sintomas depressivos.
A prevalência de mulheres que tiveram história de tratamento, de internação psiquiátrica, de uso de drogas e/ou de álcool, e, ainda, de tentativa de suicídio foi mais elevada quando estavam em liberdade e não quando na prisão. Esse dado é confirmado pelo estudo de Johnson 4 , o qual pontua que $41 \%$ das mulheres encarceradas fecharam os critérios para dependência de substância psicoativa e $73 \%$ apresentaram problemas de saúde mental ou emocional seis meses antes de serem presas. Também reforça essas conclusões outro estudo ${ }^{24}$, que salienta existirem poucas diferenças significativas entre as amostras de presas recém- ingressadas na prisão (poucos dias após a reclusão) e as presas com maior tempo no cárcere (amostra transversal). Este estudioso considera que as altas taxas de problemas de saúde mental ocorrem em um contexto de baixo desenvolvimento educacional, desemprego, problemas de moradia e um ciclo de interrupção familiar e institucionalização; fora, portanto, do ambiente prisional. Todavia, quanto maior o tempo de reclusão mais o nível da doença mental poderá ser agravado.

Os resultados do presente estudo revelam uma alta prevalência de história de violência e fuga de casa em idade precoce, e, ainda, aponta que os membros da família da presidiária apresentam uma história de uso de drogas e/ou álcool, problemas psiquiátricos e já foram recolhidos à prisão.

A violência sofrida pela mulher também é confirmada por um outro estudo 25 , o qual menciona que $70 \%$ da amostra reportaram vitimização direta. Ainda, $62 \%$ das mulheres encarceradas sofreram algum tipo de trauma infantil, sendo $48 \%$ abuso sexual 26 .

Assim, os problemas de saúde mental, manifestados pelas mulheres encarceradas, estão relacionados com sua história pregressa. Tais informações vão ao encontro do estudo de Johnson 4, no o qual fatores sociodemográficos e história pessoal estão associados com o uso de substâncias e problemas de saúde mental. Fatores associados com problemas de saúde mental incluem experiências passadas de abuso sexual ou emocional, prescrição para uso de medicação psiquiátrica e dependência de substâncias psicoativas e comorbidade de dependência de substâncias químicas e álcool.

Os dados obtidos nesta pesquisa apontam que as mulheres encarceradas que sofreram violência sexual e não-sexual apresentam maior número de sintomas depressivos do que as que não sofreram. As vítimas de violência não-sexual, com história de tratamento psiquiátrico, ideação e tentativa de suicídio e que possuem familiares com problemas psiquiátricos, têm, significativa- 
mente, maiores chances de desenvolver a sintomatologia depressiva do que as encarceradas que não possuem tais características.

A população carcerária, além de apresentar as características relacionadas acima, pode demonstrar uma saúde mental mais suscetível ao agravamento, decorrente do aprisionamento e diante de sua história passada e atual. A maioria das mulheres encarceradas entrevistadas neste estudo provém de um meio social com precárias condições socioeconômicas, como pode ser observado no baixo nível de escolaridade e profissão. Esse dado é confirmado por um estudo, segundo o qual o curso de transtornos psicológicos e comportamentais é influenciado pelo nível social e econômico do indivíduo 5 . Os resultados desta pesquisa confirmam que quanto mais escolaridade os presos possuírem melhor poderão enfrentar o estresse do aprisionamento.

Considerando-se que o presente estudo apontou que os problemas psicológicos são desencadeados quando as mulheres estão livres, considerando-se que o nível de escolaridade das presidiárias é baixo e considerando-se, ainda, que em sua grande maioria elas exercem profissões de baixo status social e/ou econômico, sugerimos como intervenções a articulação de programas de saúde mental, cursos técnicos profissionalizantes e o incentivo na inserção no mercado de trabalho, oferecido dentro da comunidade. Seria útil a continuidade de tais medidas dentro do sistema prisional para que as presas retornem à sociedade com uma melhor saúde mental.

O sistema prisional também deve ser entendido como uma possibilidade de tratamento psicoterapêutico para as detentas, articulado com a criação de programas de saúde pública. Dessa forma, os sintomas emocionais depressivos poderão ser minimizados e as presas estarão mais preparadas para retornar à vida em sociedade.

As limitações deste trabalho encontram-se principalmente no delineamento da pesquisa que não contemplou um instrumento de diagnóstico de transtornos mentais e, principalmente, de transtornos de personalidade, que poderiam fornecer um perfil mais detalhado e preciso da mulher encarcerada. Com relação à aplicação dos instrumentos, a inclusão de indivíduos sem avaliar se estes têm condições ou não de responder a um questionário (por problemas psiquiátricos, neurológicos ou baixo nível educacional) também pode ter sido uma limitação do estudo.
Além disso, os dados podem estar subestimados e a prevalência ser ainda mais elevada. Muitas participantes podem não ter relatado histórias de abusos ou ter negado o envolvimento com drogas e álcool, com receio de que a pesquisa pudesse lhes causar algum prejuízo em relação à sua situação jurídica - a amostra de prisioneiros apresenta certas peculiaridades que podem interferir em resultados mais fidedignos. Neste estudo, mais da metade da amostra era de presas provisórias, que estão respondendo ao processo criminal e aguardando a investigação final. Já a outra parte, que são as mulheres condenadas, passa por uma avaliação final, durante a qual muitas vezes é solicitada uma avaliação psicológica judicial para verificar se os apenados já estão preparados para retornar à vida em sociedade. Embora tenham sido esclarecidos os reais objetivos, algumas mulheres podem ter associado que este estudo interferiria no processo investigativo, que compreende desde o ingresso no sistema prisional até o término do cumprimento da pena.

\section{Considerações finais}

A estimativa da gravidade dos problemas de saúde mental nas encarceradas, por meio de uma alta prevalência de sintomas depressivos e envolvimento com drogas e álcool, denota que a história de vida marcada por alguns fatores estressores contribuiu para o desencadeamento de tais sintomatologias.

Um dado importante é que os sintomas depressivos e o envolvimento com drogas não são desencadeados pelo aprisionamento, mas sim durante a vida em liberdade. Em verdade, diversos são os fatores que podem ser associados à prevalência dos referidos problemas mentais, dentre eles, problemas sociodemográficos, baixa renda, problemas clínicos, histórico familiar conturbado.

Esses achados indicam a necessidade de uma atenção multidisciplinar para as mulheres presas, sempre integrando diversas modalidades de tratamento (psicológico, psiquiátrico, terapia ocupacional). Seria útil, ainda, um maior investimento no sistema prisional, no sentido de ampliar e qualificar os serviços de saúde mental, com o intuito de fornecer a essa parcela da população um tratamento adequado, frente a todas as características apresentadas neste estudo. 


\section{Resumo}

O estudo objetivou traçar o perfil, descrever as características sociodemográficas e clínicas da mulher prisioneira, além de verificar a prevalência de sintomas depressivos e de desesperança, uso, abuso e dependência de drogas e álcool. Participaram 287 mulheres de uma Penitenciária Feminina do Estado do Rio Grande do Sul, Brasil. O delineamento foi de um estudo quantitativo e transversal. Os achados mostram que o perfil caracteriza-se por ser solteira, jovem, ter no mínimo dois filhos, ter exercido atividades informais e geralmente de baixo status social elou econômico, possuir até o Ensino Fundamental incompleto. Foi encontrada alta prevalência de sintomas depressivos e uso, abuso e dependência de drogas, porém baixa prevalência de sintomas de desesperança. Diversos fatores, principalmente ligados à vida pregressa das participantes, foram associados significativamente com os sintomas depressivos.

Prisioneiros; Mulheres; Depressão; Drogras Ilícitas

\section{Colaboradores}

D. Canazaro e I. I. L. Argimon participaram da concepção do estudo; aquisição, análise e interpretação dos dados; da redação e revisão crítica o artigo, além da aprovação da versão final do texto.

\section{Agradecimentos}

À Coordenação de Aperfeiçoamento de Pessoal de Nível Superior (CAPES) e ao Conselho Nacional de Desenvolvimento Científico e Tecnológico (CNPq) pelo financiamento.

\section{Referências}

1. Cerqueira D, Lobão W. Determinantes da criminalidade: arcabouços teóricos e resultados empíricos. Dados Rev Ciênc Sociais 2004; 47:233-69.

2. Assadi SM, Noroozian M, Pakravannejad M, Yahyazadeh O, Aghayan S, Shariat SV, et al. Psychiatric morbidity among sentenced prisoners: prevalence study in Iran. Br J Psychiatry 2006; 188: 159-64.

3. Tye SC, Mullen EP. Mental disorders in female prisoners. Aust N Z J Psychiatry 2006; 40:266-71.

4. Johnson H. Concurrent drug and alcohol dependency and mental health problems among incarcerated women. Aust N Z J Criminol 2006; 39:190217.

5. Narkauskaité L, Juozulynas A, Mackiewicz Z, Surkiene G, Prapiestis J. The prevalence of psychotropic substance use and its influencing factors its influencing factors in Lithuanian penitentiaries. Med Sci Monit 2007; 13:131-5.

6. Butler T, Allnutt S, Cain D, Owens D, Muller C. Mental disorder in the New South Wales prisoner population. Aust N Z J Psychiatry 2005; 39:407-13.
7. Gunter DT, Arndt S, Wenman G, Allen J, Loveless $\mathrm{P}$, Sieleni B, et al. Frequency of mental and addictive disorders among 320 men and women entering the Iowa prison system: use of the MINI-Plus. J Am Acad Psychiatry Law 2008; 36:27-34.

8. Australian Bureau of Statistics. Mental health and wellbeing profile of adults. Canberra: Australian Bureau of Statistics; 1997.

9. Blaauw E, Roesch R, Kerkhof A. Mental disorders in European prison systems: arrangements for mentally disordered prisoners in the prison systems of 13 European coutries. Int J Law Psychiatry 2000; 23:649-63.

10. Trestman RL, Ford J, Zhang W, WiesbrockV. Current and lifetime psychiatric illness among inmates not identified as acutely mentally ill at intake in Connecticut's jails. J Am Acad Psychiatry Law 2007; 35:490-500.

11. Lewis C. Treating incarcerated women: gender matters. Psychiatr Clin North Am 2006; 29:773-89. 
12. Fazel S, Bains P, Doll H. Substance abuse and dependence in prisoners: a systematic review. Addiction 2006; 101:181-91.

13. Fazel S, Danesh J. Serious mental disorder in 23000 prisoners: a systematic review of 62 surveys. Lancet 2002; 359:545-50.

14. Women in Prison. Women prisoners. http://www. womeninprison.org.uk/womenprisoners.php (acessado em 10/Set/2008).

15. Covington SS. Women and the criminal justice system. Womens Health Issues 2007; 17:180-2.

16. Moraes PAC, Dalgalarrondo P. Mulheres encarceradas em São Paulo: saúde mental e religiosidade. J Bras Psiquiatr 2006; 55:50-6.

17. Staton M, Leukefeld C, Webster JW. Substance use, health: problems and service utilization among incarcerated women. Int J Offender Ther Comp Criminol 2003; 47:224-39.

18. Chapman LA, Specht WM, Cellucci T. Factors associated with suicide attempts in female inmates: the hegemony of hopelessness. Suicide Life Threat Behav 2005; 35:558-68.

19. von Schönfeld CE, Schneider F, Schröder T, Widmann B, Botthof U, Driessen M. Prävalenz psychischer störungen, psychopathologie und behandlungsbedarf bei weblichen und männlichen gefangenem. Nervenarzt 2006; 77:830-41.
20. Michaud P. Les problems d'alcool chez les détenus. Alcoologie et Addictologie 2006; 28:375-85.

21. Watzke S, Ullrich S, Marneros A. Gender- and violence- related prevalence of mental disorders in prisoners. Eur Arch Psychiatry Clin Neurosci 2006; 256:414-21.

22. Belenko S, Peught J. Estimating drug treatment needs among state prison inmates. Drug Alcohol Depend 2005; 77:269-81.

23. Daigle MS, Côté G. Nonfatal suicide: related behavior among inmates: testing for gender and type differences. Suicide Life Threat Behav 2006; 36:670-81.

24. Wright B, Duffy D, Curtin K, Linehan S, Monks S, Kennedy GH. Psychiatric morbidity among women prisoners newly committed and amongst remanded and sentenced. Ir J Psychol Med 2006; 23:47-53.

25. Harris RM, Sharps PW, Allen K, Anderson EH, Soeken K, Rohatas A. The interrelations between violence, HIV/AIDS, and drug use in incarcerated women. J Assoc Nurses AIDS Care 2003; 14:27-40.

26. Green BL, Miranda J, Daroowalla A, Siddique J. Trauma exposure, mental health functioning, and Program Needs of Women in Jail. Crime Delinq 2005; 51:142-51.

Recebido em 06/Abr/2009

Versão final reapresentada em 07/Nov/2009

Aprovado em 24/Mai/2010 